\title{
Global neurosurgery: the current capacity and deficit in the provision of essential neurosurgical care. Executive Summary of the Global Neurosurgery Initiative at the Program in Global Surgery and Social Change
}

\author{
Michael C. Dewan, MD, MSCl,1,2 Abbas Rattani, MBe, ${ }^{1,3}$ Graham Fieggen, MD, MSc, ${ }^{4}$ \\ Miguel A. Arraez, MD, PhD, ${ }^{5}$ Franco Servadei, MD, ${ }^{6}$ Frederick A. Boop, MD, ${ }^{7}$ \\ Walter D. Johnson, MD, MBA, MPH, ${ }^{8}$ Benjamin C. Warf, MD, ${ }^{9,10}$ and Kee B. Park, MD ${ }^{1}$ \\ ${ }^{1}$ Global Neurosurgery Initiative-Program in Global Surgery and Social Change, Department of Global Health and Social \\ Medicine, Harvard Medical School, Boston, Massachusetts; '2Department of Neurological Surgery, Vanderbilt University \\ Medical Center, Nashville, Tennessee; ${ }^{3}$ Meharry Medical College School of Medicine, Nashville, Tennessee; ${ }^{4}$ Department of \\ Surgery, University of Cape Town, South Africa; ${ }^{5}$ Department of Neurosurgery, Carlos Haya University Hospital, Malaga, Spain; \\ ${ }^{6}$ Department of Neurosurgery, Humanitas University and Research Institute, Milan, Italy; 'Department of Neurological Surgery, \\ University of Tennessee Health Sciences Center, LeBonheur Children's Hospital Neurosciences Institute, Semmes-Murphey \\ Clinic, Memphis, Tennessee; ${ }^{8}$ Emergency \& Essential Surgical Care Programme Lead, World Health Organization, Geneva, \\ Switzerland; ' Department of Neurological Surgery, Boston Children's Hospital, Harvard Medical School, Boston, Massachusetts; \\ and ${ }^{10}$ CURE Children's Hospital of Uganda, Mbale, Uganda
}

OBJECTIVE Worldwide disparities in the provision of surgical care result in otherwise preventable disability and death. There is a growing need to quantify the global burden of neurosurgical disease specifically, and the workforce necessary to meet this demand.

METHODS Results from a multinational collaborative effort to describe the global neurosurgical burden were aggregated and summarized. First, country registries, third-party modeled data, and meta-analyzed published data were combined to generate incidence and volume figures for 10 common neurosurgical conditions. Next, a global mapping survey was performed to identify the number and location of neurosurgeons in each country. Finally, a practitioner survey was conducted to quantify the proportion of disease requiring surgery, as well as the median number of neurosurgical cases per annum. The neurosurgical case deficit was calculated as the difference between the volume of essential neurosurgical cases and the existing neurosurgical workforce capacity.

RESULTS Every year, an estimated 22.6 million patients suffer from neurological disorders or injuries that warrant the expertise of a neurosurgeon, of whom 13.8 million require surgery. Traumatic brain injury, stroke-related conditions, tumors, hydrocephalus, and epilepsy constitute the majority of essential neurosurgical care worldwide. Approximately 23,300 additional neurosurgeons are needed to address more than 5 million essential neurosurgical cases-all in lowand middle-income countries-that go unmet each year. There exists a gross disparity in the allocation of the surgical workforce, leaving large geographic treatment gaps, particularly in Africa and Southeast Asia.

CONCLUSIONS Each year, more than 5 million individuals suffering from treatable neurosurgical conditions will never undergo therapeutic surgical intervention. Populations in Africa and Southeast Asia, where the proportion of neurosurgeons to neurosurgical disease is critically low, are especially at risk. Increasing access to essential neurosurgical care in low- and middle-income countries via neurosurgical workforce expansion as part of surgical system strengthening is necessary to prevent severe disability and death for millions with neurological disease.

https://thejns.org/doi/abs/10.3171/2017.11.JNS171500

KEYWORDS capacity; epidemiology; global; incidence; volume; workforce; worldwide

ABBREVIATIONS HICs = high-income countries; LMICs = low and middle-income countries; PGSSC = Program in Global Surgery and Social Change; TBI = traumatic brain injury; TSI = traumatic spinal injury; WFNS = World Federation of Neurosurgical Societies.

SUBMITTED June 21, 2017. ACCEPTED November 10, 2017.

INCLUDE WHEN CITING Published online April 27, 2018; DOI: 10.3171/2017.11.JNS171500. 
I N 2015, the Lancet Commission on Global Surgery offered a summary of the surgical burden and described existing gaps in the provision of safe and affordable surgical care worldwide. ${ }^{11}$ More than two-thirds of the world's population lack access to appropriate surgical and anesthetic care, equating to an estimated 143 million necessary surgical procedures that are left undone. This untreated surgical disease results in extreme economic costs and profound disability and death. ${ }^{17}$

Within this tremendous burden of surgical disease resides the contribution of neurosurgical disease. Obtaining a reliable estimate of the volume of neurosurgical disease requires addressing numerous challenges including sparse epidemiological data, heterogeneous literature reporting, and even competing definitions of disease entities. Furthermore, quantifying the existing workforce of surgeons capable of safely addressing neurological disease is difficult. There exists neither a single worldwide registry of neurosurgeons, nor even a consensus as to the requisite training and competencies of a neurosurgeon.

Despite debate about the nature of neurosurgical disease and those capable of addressing it, ${ }^{1}$ there is growing recognition of a worldwide shortage of neurosurgeons, particularly in low-resourced settings. ${ }^{2,13,20}$ Before pursuing a targeted campaign to stem the disparity in specialized surgical care, a more definitive understanding of the problem is essential, including regions with the greatest need. In this report we summarize a body of research by the Program in Global Surgery and Social Change aimed at establishing the current status of neurosurgery worldwide. Thus, we provide estimates of the global volume of neurosurgical disease, the existing neurosurgical capacity, and the current unmet neurosurgical need worldwide.

\section{Methods}

\section{Volume of Neurosurgical Disease}

We began by estimating the incidence of 10 conditions encountered by neurosurgeons that form the foundation of essential neurosurgical care: brain and spinal tumors, hydrocephalus, traumatic brain injury (TBI), traumatic spinal injury (TSI), neural tube defects, stroke, CNS vascular anomalies, CNS infections, and epilepsy. Conditions were identified by ranking commonly treated neurosurgical conditions described by an international panel of neurosurgical providers (see Proportion Requiring Neurosurgery below). Then, those conditions in which treatment neglect would directly result in severe disability or death were designated by this report as essential neurosurgical conditions. Notably excluded from the final list are degenerative spine disease and osteoporotic vertebral fractures. ${ }^{15}$ The proportion and volume of patients succumbing to severe disability or death if these conditions are not treated surgically is difficult to determine, particularly in resource-limited settings. Due to the recognition of limited capacity, this classification scheme of essential neurosurgical care also excluded important conditions such as pain disorders, spasticity, movement disorders, and other conditions for which neurosurgical treatment might offset disability and lost productivity. Competency with the treatment of the 10 conditions above, however, would equip a neurosurgeon anywhere with the ability to treat the vast majority of patients with neurosurgical disease, and thereby most efficiently avert severe disability or premature death.

Reliable epidemiological figures were available for two diseases (neural tube defect and stroke; http://ghdx. healthdata.org/gbd-results-tool), ${ }^{10}$ thus our attention was focused on the remaining conditions. National surgical registries were available for brain and spinal tumors allowing region-specific estimation of disease incidence. ${ }^{3}$ The Institute for Health Metrics and Evaluation has published epidemiological figures for road traffic injuries in each country, which were used to extrapolate the incidence of TBI. The proportion of traffic collisions resulting in TBI was calculated via a meta-analysis of published traffic injury data. Similarly, the proportion of TBI from which traffic collisions is the mechanism of action was calculated via a meta-analysis. Applying these ratios to the incidence of road traffic injuries delivered an estimation of total TBI from all causes. ${ }^{6}$

For the remaining conditions (hydrocephalus, TSI, CNS vascular anomalies, CNS infections, and epilepsy), an exhaustive systematic review was conducted to identify studies reporting epidemiological data from large, population-based cohorts. After extracting case numbers and sample size, a meta-analysis was performed to estimate disease incidence figures for each disease by WHO region and by World Bank income group. Finally, incidence figures were multiplied by population estimates from the WHO to generate estimates for the annual volume of disease globally. Detailed methodology and results are de-

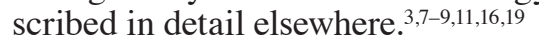

\section{Proportion Requiring Neurosurgery}

Identifying the incidence of neurosurgical disease requires an estimate of the proportion of neurological injury or illness warranting surgical intervention. Some neurosurgical diseases are best managed operatively, while others require nonoperative neurosurgical management. Some neurological conditions do not require neurosurgical expertise for optimal treatment. While surgical logs from ideal health care systems offer reliable estimates for surgical proportion (i.e., the fraction of neurological conditions requiring surgery), their relevance may not be generalizable to health care systems in differing settings. Furthermore, we learn very little about the proportion of disease benefiting from nonoperative neurosurgical expertise or consultation. Thus, following the precedent established by the Lancet Commission on Global Surgery, we sought expert opinion from a broad, diverse group of surgical providers.

Via electronic survey, nearly 200 neurosurgeons from more than 50 countries were asked to estimate the proportion of specific neurological disorders that, in an ideal world, would require either neurosurgical operation or consultation. To gauge degree of certainty, surgeons indicated how confident they were in their estimation for each disease. Finally, we sought to identify the number of cases that can be reasonably performed each year by the average practicing neurosurgeon. Surgeons indicated their annual case volume and types of cases most frequently 
performed, then indicated how they perceived their workload at that clinical volume. The full questionnaire and analytical methodology are described in detail elsewhere. ${ }^{5}$

\section{Neurosurgical Workforce}

Determination of the current neurosurgical workforce was quantified via a large, multinational global mapping project facilitated by the World Federation of Neurosurgical Societies (WFNS), the WHO, and the Program in Global Surgery and Social Change (PGSSC) at Harvard Medical School. Briefly, Ministries of Health, officers of the 130 societies registered with the WFNS, and the members of the WHO Global Initiative for Emergency and Essential Care were contacted via email for information. For some countries an internet search was conducted to identify the contact information for active neurosurgeons willing and able to complete an online questionnaire on capacity. Surgeons registered with the open access Global Neurosurgery forum (https://globalneurosurgery.org/ members) were also included. The data collection instrument captured surgeon numbers and geographic locations, as well as the presence of basic equipment germane to neurosurgical care: high-speed drills, bipolar electrocautery, imaging infrastructure, etc. For the purposes of the current summary, we aggregated the number of neurosurgeons by country and by WHO region to facilitate quantification of the current surgical capacity. A comprehensive description of the methodology and results of the PGSSC workforce mapping initiative is described elsewhere (unpublished data). ${ }^{14}$

\section{Estimating the Deficit in Neurosurgical Care}

The unmet need, or deficit in neurosurgical care, represents the difference between the total estimated number of essential neurosurgical conditions presenting for treatment and the current number of neurosurgical cases that can be performed given the existing workforce. The number of essential neurosurgical conditions was calculated by taking the product of neurosurgical volume (from each metaanalysis by disease entity $3,7-9,11,16,19)$ and surgical proportion (from the provider survey ${ }^{5}$ ). The number of neurosurgical cases that can be currently performed, on the other hand, was obtained by taking the product of the number of neurosurgeons (from the Workforce estimate [unpublished data]) and the median number of cases performed (from the provider survey estimate ${ }^{5}$. The deficit was then calculated on a regional and income-group basis to provide a more granular description of need and volume, and to highlight disparity in neurosurgical care across regions. Then, a country-specific ratio of neurosurgeons to essential neurosurgical cases (both surgical and consultative) was estimated. The total number of cases within a region was allocated to each country according to the population proportion of a given country within its WHO region and World Bank income group. For the worldwide estimate, all countries with available workforce data were included, irrespective of WHO or World Bank classification. Incorporating the country-specific neurosurgeon figure from the workforce survey, maps were then created to depict this ratio using Tableau software.

\section{Results}

Estimations of the volume of each disease, the proportion requiring neurosurgical intervention, and the quantification of the existing neurosurgical workforce have been described in detail elsewhere. Here, we have aggregated this information and synthesized the findings into a digestible summary.

\section{Neurosurgical Cases by Region and Income Group}

An estimated 13.8 million essential neurosurgical cases develop each year, of which more than $80 \%$ arise in low- and middle-income countries (Table 1). A detailed breakdown of neurosurgical volume by disease can be found in the Appendix (Tables S1-S23). Corresponding to the regional population proportions, an expected 3.5 million and 3.7 million new cases are expected in Southeast Asia and the Western Pacific, respectively. Africa is expected to endure nearly 2 million neurosurgical cases, in contrast to about 665,000 cases in the US and Canada. Europe, the Eastern Mediterranean, and Latin America each will encounter between 1.1 and 1.8 million new neurosurgical cases annually (Table 1). Among the conditions we have defined as requiring essential neurosurgical care, surgery for TBI (burr holes, craniotomy/craniectomy, etc.) accounts for $45 \%$, cerebrovascular accident for $20 \%$, hydrocephalus for $7 \%$, and brain tumors for $5 \%$. Vascular anomalies $(2.2 \%)$, neural tube defects $(0.3 \%)$, and spinal tumors $(0.1 \%)$ occupy a relatively modest proportion of the global neurosurgical need.

The number of cases requiring neurosurgical consultation-but not necessarily surgical intervention-is far greater, approaching 22.6 million annually (Table 2). Again, Southeast Asia and the Western Pacific bear the greatest consultative demand with 5.8 million and 6.2 million cases each year, respectively. Low and middle-income countries (LMIC) endure the greatest burden at 17.6 million cases, compared with 4.3 million cases in high-income countries (HIC).

\section{Neurosurgeons by Region and Income Group}

We identified 49,940 practicing neurosurgeons worldwide (unpublished data). Ninety-eight percent (98.9\%) of neurosurgeons were identified via the WFNS-sponsored electronic survey, while 560 neurosurgeons $(1.1 \%)$ from 34 countries $(14.9 \%)$ in which no data were available were imputed via statistical modeling. The greatest population of neurosurgeons resides in the Western Pacific region, with China and Japan alone accounting for more than 18,000 neurosurgeons. Europe and the US/Canada have the next largest populations of neurosurgeons, with 10,719 and 5296 neurosurgeons, respectively. In the African region, with a population of 990 million, 488 neurosurgeons were identified (Table 3). Forty-four percent (44\%) of neurosurgeons worldwide reside in high-income countries.

Previously, we have identified the mean neurosurgical volume reasonably managed by a neurosurgeon in a given country as 223 cases per year. ${ }^{5}$ This assumes a surgeon's perceived workload is maintained at a level of 75 out of 100 (where 0 is "not at all busy" and 100 is "extremely busy, overworked"). The greatest total neurosurgical ca- 


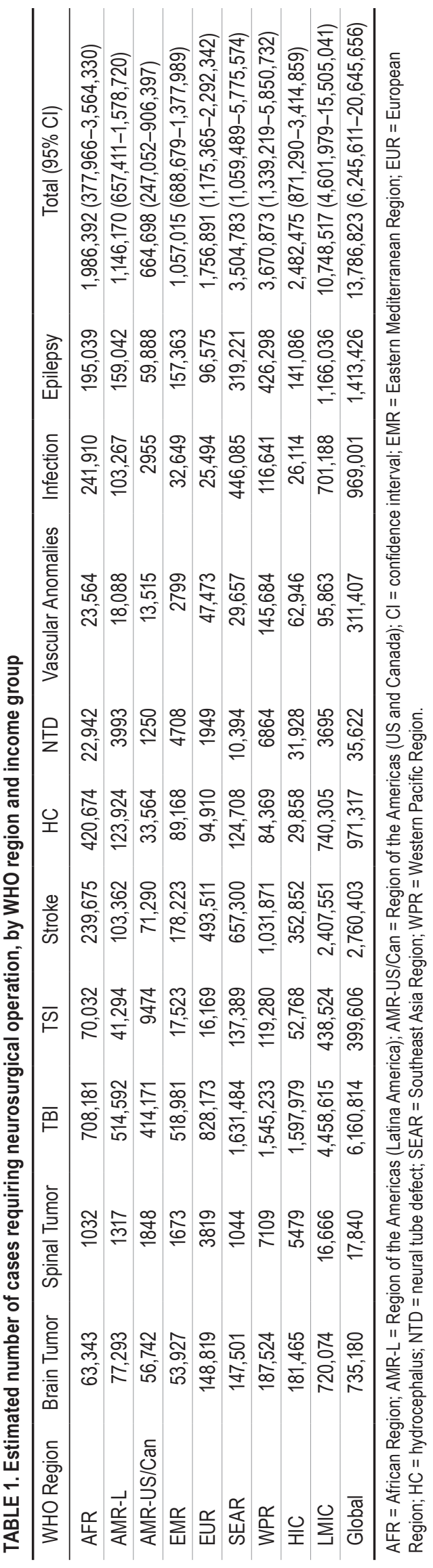

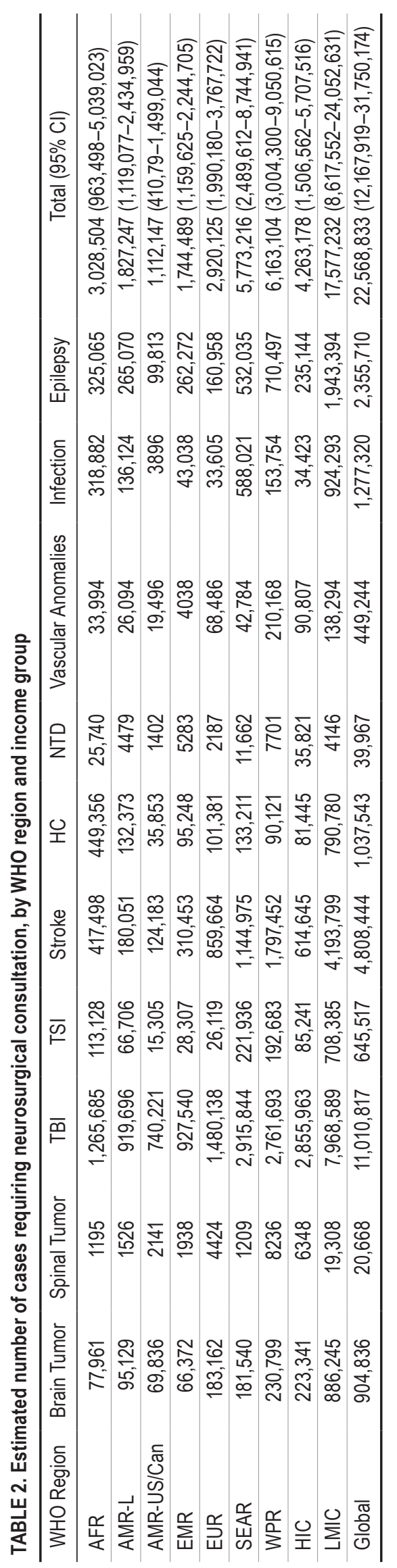


TABLE 3. Summary of case volume, surgical capacity, and neurosurgical deficit

\begin{tabular}{lccccc}
\hline WHO Region & Case Volume & Current Surgeons & Case Capacity & Case Deficit* & \multicolumn{2}{c}{ Additional Surgeons Necessary* $^{*}$} \\
\hline AFR & $1,986,392$ & 488 & 108,824 & $1,877,568$ & 8420 \\
\hline AMR-L & $1,146,170$ & 4216 & 940,168 & 206,002 & 924 \\
\hline AMR-US/Can & 664,698 & 5296 & $1,181,008$ & - & - \\
\hline EMR & $1,057,015$ & 2073 & 462,279 & 594,736 & 2667 \\
\hline EUR & $1,756,891$ & 10,719 & $2,390,337$ & - & - \\
\hline SEAR & $3,504,783$ & 4409 & 983,207 & $2,521,576$ & 11,308 \\
\hline WPR & $3,670,873$ & 22,114 & $4,931,422$ & - & - \\
\hline Worldwide & $13,786,823$ & 49,940 & $11,136,620$ & $5,199,883$ & 23,318 \\
\hline
\end{tabular}

* "Case Deficit" and "Additional Surgeons Necessary" are left blank for regions that have a capacity surplus. Capacity surplus suggests cases are being performed by neurosurgeons that fall outside of the definition of "essential neurosurgical care," such as degenerative spine disease and movement disorders.

pacity exists in the Western Pacific region, where more than 22,000 neurosurgeons are capable of performing nearly 5 million essential neurosurgical cases annually. In contrast, the nearly 500 neurosurgeons in Africa can be expected to perform approximately 110,000 neurosurgical operations. Worldwide, and ignoring geographic boundaries and uneven case distribution, the overall surgical capacity is approximately 11 million essential neurosurgical cases per year (Table 3).

\section{The Deficit in Neurosurgical Care}

The current deficit in neurosurgical care is approximately 5.2 million (Table 3). This deficit derives from the difference between the number of essential cases encountered annually and the essential surgical capacity, where essential surgical capacity excludes nonessential cases performed by neurosurgeons, particularly in HIC. To meet this demand, an estimated additional 22,626 neurosurgeons would need to be trained. The greatest deficit resides in Southeast Asia where nearly 2.5 million essential cases go unmet. The tremendous shortage of neurosurgeons in India-where 3500 neurosurgeons are responsible for a population of more than 1.2 billion-is largely responsible for this health care discrepancy. For the entire region, 2.5-times the existing number of surgeons are needed to provide appropriate coverage. In Africa, where more than 1.8 million cases are not being addressed by capable neurosurgeons, the surgical workforce must increase by more than $1700 \%$ to meet the regional demand. The vast majority of the population in Africa has no access to a neurosurgeon, independent of other geographic, social, or financial barriers that may exist. ${ }^{14}$ In terms of essential care, three WHO regions-the region of the Americas restricted to the US and Canada only, the European region, and the Western Pacific region-do not demonstrate a workforce deficit, although the need for additional neurosurgeons in these regions to meet neurological conditions not accounted for in our analysis remains a salient consideration.

\section{Discussion}

In this summary, for neurosurgical diseases, we estimate approximately 13.8 million new operative cases and 22.6 million new consultative cases exist worldwide each year. TBI and stroke-related conditions and sequelae constitute approximately $60 \%$ of essential neurosurgical volume, with tumors, hydrocephalus, epilepsy, and infectious-related conditions accounting for the majority of the remaining proportion of disease. A tremendous disparity exists in the global workforce; $82 \%$ of neurosurgical volume exists in LMICs where approximately $56 \%$ of neurosurgeons reside. While Africa accounts for $15 \%$ of the global volume of neurosurgical disease, African hospitals and health care networks have access to less than $1 \%$ of the neurosurgeon community. An estimated 23,300 additional surgeons - approximately 11,300 in Southeast Asia, and 8400 in Africa-are needed in low- and middleincome countries to adequately address essential neurosurgical disease worldwide. For the first time, an estimate of the deficit of neurosurgical care is provided. The world maps (Figs. 1 and 2) offer a pictorial representation of the volume of essential neurosurgical disease observed by the existing neurosurgical workforce in a given country; disparity in care is illustrated. Training capable and competent neurosurgeons is a fundamental step in establishing equal access to safe and timely neurosurgical care. A benchmark is thus established for which to aim.

Examining Tables 1 and 2 more closely, there are $3 \mathrm{im}$ portant observations that merit specific discussion. First, these disease figures are estimates. While they derive from the best available evidence and are computed using modern statistical modeling, they remain susceptible to the same methodological assumptions made for any epidemiological estimation of disease incidence. Second, we notice that diseases requiring advanced diagnostic equipment for diagnosis (e.g., MRI) are more likely to be underrepresented in regions made up of predominantly low income or lower middle income countries. CNS tumors are taken as an example. There exists a positive correlation between age and incidence of brain tumors. Most populations in HICs exhibit a greater median survival than those in LMICs. Thus, ignoring competing variables (such as carcinogenic exposures, or prevention of metastatic spread) finding a higher proportion of brain tumors in the US/Canadian region than in African region follows logic. However, the degree to which this difference exists suggests additional factors are involved. For these less clinically overt diseases, a diagnosis bias almost certainly affects these results. Brain and spinal tumors in low-resource settings are not being 


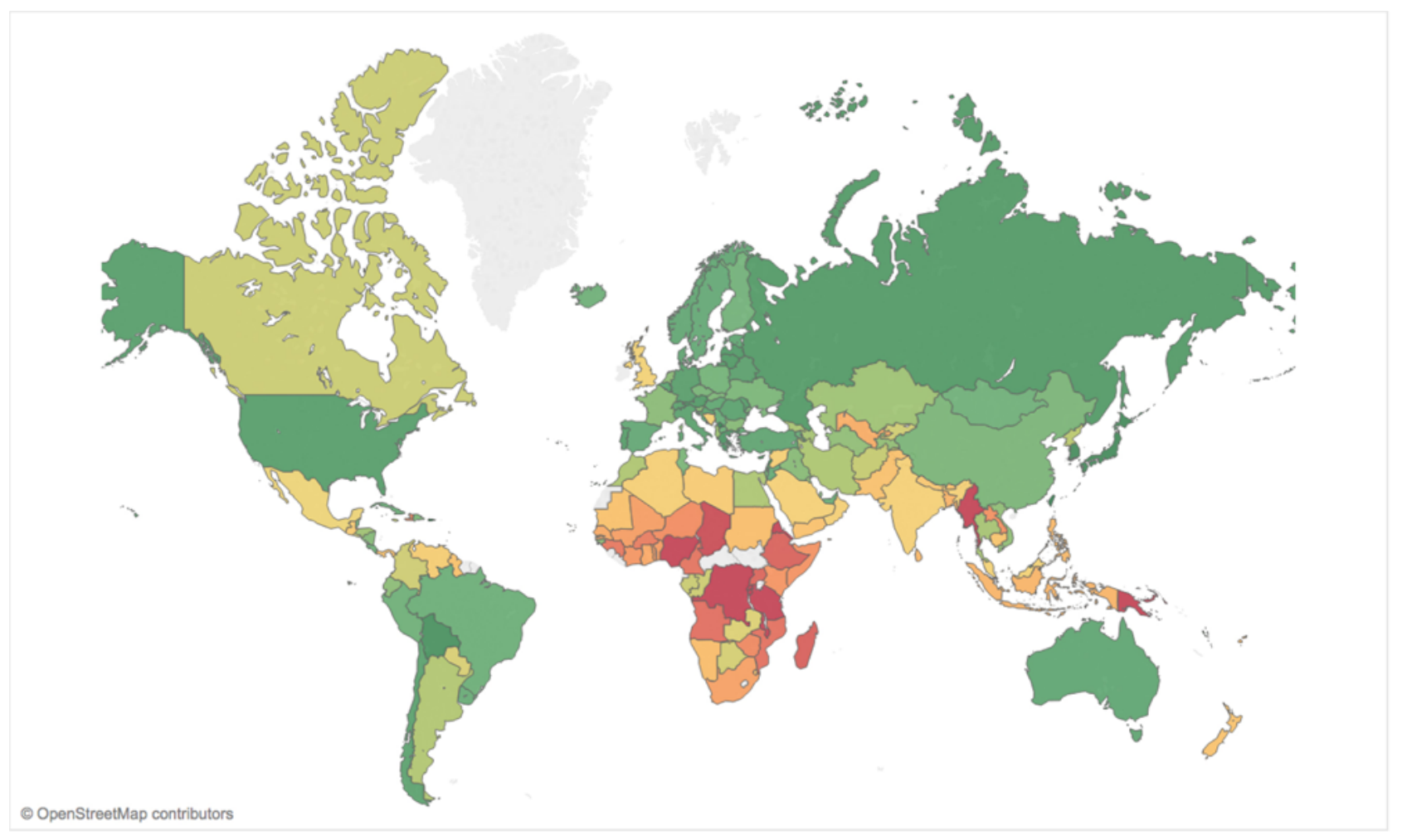

Annual essential surgical cases per neurosurgeon

500

FIG. 1. The global deficit of neurosurgical care: operations. @OpenStreetMap contributors (http://www.openstreetmap.org/ copyright). Figure is available in color online only.

diagnosed due to lack of equipment and/or lack of access to the level of care requisite for their diagnosis. Conversely, readily diagnosed diseases such as neural tube defects are more likely to be captured by population-based epidemiological studies, regardless of diagnostic infrastructure. Third, while the estimates are staggering-especially in relation to the existing workforce-they are most certainly an underestimate of the true surgical responsibilities faced by the global neurosurgery community. Absent from these estimates are entities such as diagnostic procedures (lumbar punctures, ventricular taps, etc.), repeat or redo surgeries, and, importantly, the great proportion of neurosurgical interventions that are deemed "nonessential" by the outlined criteria.

This year, nearly 5.2 million essential neurosurgical cases are estimated to go unmet by a qualified neurosurgeon, resulting in otherwise avoidable disability and death. Such essential neurosurgical cases include relatively straightforward procedures such as burr hole craniotomy for hematoma evacuation and shunt insertion for childhood hydrocephalus. Interestingly, these are the only two surgeries mentioned in the 44 essential surgical procedures listed within the Disease Control Priorities series published by the World Bank Group. ${ }^{4}$ However, extraaxial hemorrhage and hydrocephalus represent only a small fraction of neurosurgical conditions that result in severe disability and death if left untreated. Essential operations as outlined in this report also include brain tumor resection, cerebral aneurysm exclusion, and open spinal fracture reduction and fixation, all considered to require more advanced surgical technique and resources. Designating treatment tiers to balance access to care and quality of delivery is imperative to optimize geographic coverage. While a discussion of this concept reaches beyond the scope of this summary, the centralization of highly complex neurosurgical carewhile also prioritizing basic, emergency neurosurgical care peripherally-is an important model that must remain a priority for the global neurosurgery community, especially in LMICs.

\section{Ongoing Efforts to Increase Neurosurgical Capacity}

A number of efforts to increase neurosurgical capacity in LMICs have already begun to make a difference. Enabling nonneurosurgeons to perform neurosurgical interventions (task-shifting) that are technically straightforward and have a high benefit to risk ratio (such as emergency evacuation of an epidural hematoma) is rational. Likewise, teaching nonneurosurgeons to place shunts for hydrocephalus has been advocated by some, but infant hydrocephalus is not immediately life-threatening; although placing the initial shunt is not difficult, the significant complication rate requires timely neurosurgical expertise for subsequent management. The ultimate goal should be 


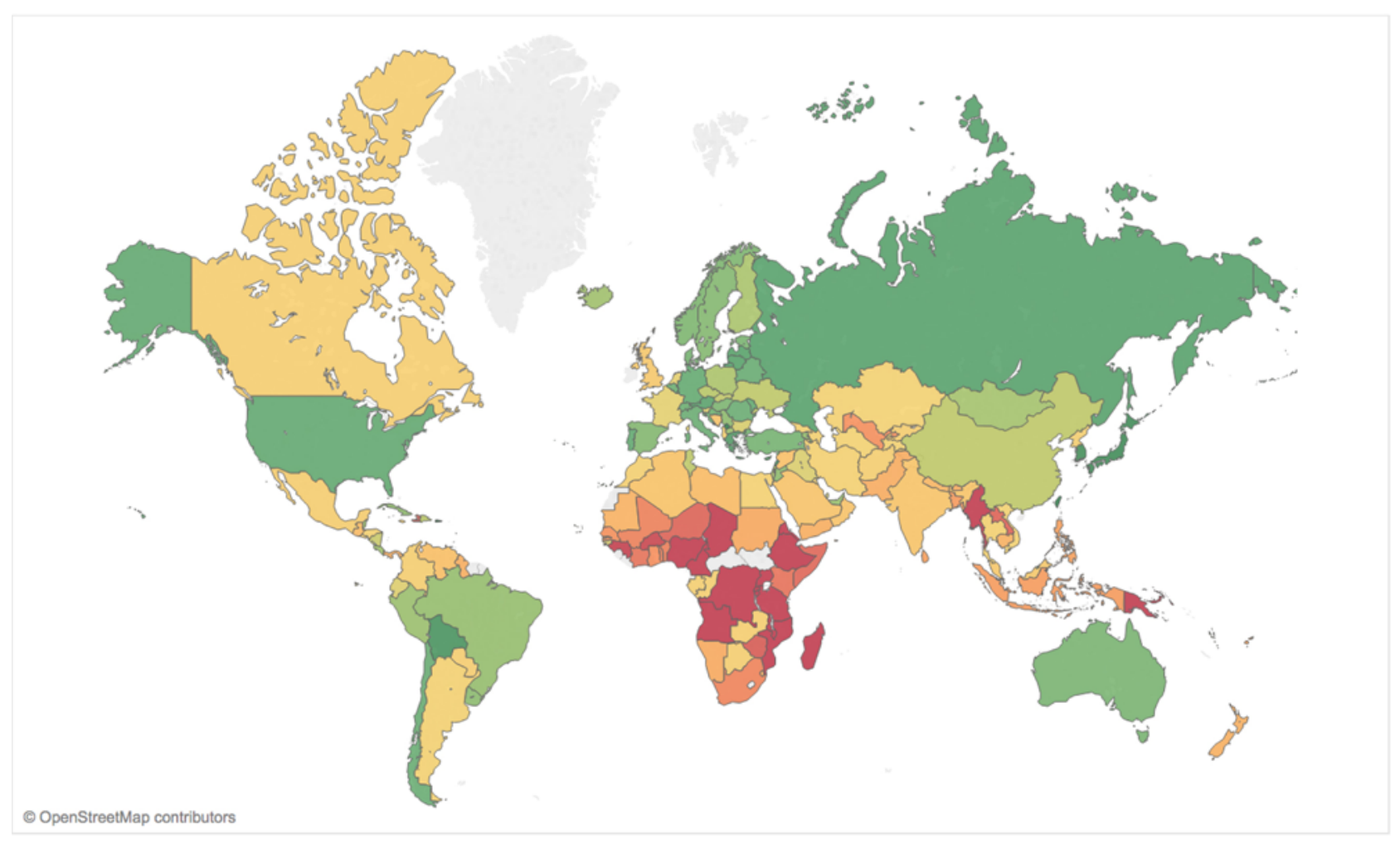

\section{Annual essential case consults per neurosurgeon}

FIG. 2. The global deficit of neurosurgical care: consultations. @OpenStreetMap contributors (http://www.openstreetmap.org/ copyright). Figure is available in color online only.

to increase neurosurgical capacity by increasing the number of competent and accessible neurosurgeons in underserved regions. The most sustainable way to achieve this will be for adequately trained national neurosurgeons to begin training programs in their home countries. This may initially require the importation of expertise, but the need for this would diminish over time if an investment is made in training individuals from these countries.

The global neurosurgery community should prioritize the provision of training to individuals from countries that do not have adequate neurosurgery residency programs. Historically, people who leave home to train in a high-income country often fail to return. But in the past decade, sub-Saharan Africa has benefitted from an influx of neurosurgeons because the few established training programs on the continent have embraced the opportunity to incorporate trainees from the less developed countries into their residency programs. Programs in Morocco, Senegal, Zimbabwe, and South Africa have trained approximately 60 new neurosurgeons from other African countries thus far. Also, a new East African training program has recently been established in cooperation with The College of Surgeons of East Central and Southern Africa, with training sites in Tanzania, Uganda, and Kenya. All these programs provide more appropriately contextualized training in regard to pathology and resource availability, and are more likely to see their trainees return home to build neurosurgi- cal capacity in their own countries. In addition, longitudinal partnerships between centers in LMICs and academic neurosurgery departments in HICs (Duke University, University of Alabama, University of Miami, The University of Toronto, The Weill Cornell Medical College, The Barrow Neurological Institute, and others) are fostering continued training, mentoring, and collaborative research in sub-Saharan Africa, Southeast Asia, and Latin America. Other programs, such as CURE Hydrocephalus and Spina Bifida, provide subspecialty training fellowships to neurosurgeons from across the developing world. Furthermore, organized neurosurgery (such as the Foundation for International Education in Neurosurgery, the WFNS, the Asian Australasian Society of Neurological Surgeons, the American Association of Neurological Surgeons, the European Society of Pediatric Neurosurgery, and the International Society of Pediatric Neurosurgery) has long been engaged in international neurosurgery education. The past 10 years have seen a noticeable escalation in awareness of and commitment to global neurosurgery that should, if we stay on mission, gradually improve access to neurosurgical care for people everywhere.

\section{The Way Forward}

The Role of Professional/National Societies

National neurosurgical societies exist to represent their 
community of neurosurgeons via surgical education, the promotion of scientific exchange, establishment of practice guidelines, and at times the promotion of a legislative agenda. For an individual surgeon, particularly one who operates in a solo practice, the national society serves as the most direct conduit to peer evaluation and feedback, continuing surgical education, and surgeon fellowship. A robust and active professional society serves to enhance the quality of neurosurgical care delivery by its membership. Thus, as the global neurosurgery effort matures, professional societies are a natural springboard from which to launch targeted intervention strategies, and may have broader impact than simply targeting a specific hospital, university, or institution.

Naturally, a professional society's role depends upon the resources and capabilities that exist on the ground. For example, in low-resource settings, high-priority initiatives of a neurosurgical society may include surveying regional disease burden, organizing available manpower, and lobbying for federal support. Meanwhile, societies in resource-rich countries can invite meaningful participation from LMIC surgeons, organize a workforce of members interested in cross-national education and training, and allocate activities, meetings, and funding to international education and research initiatives. Indeed, some societies in HICs are already playing an active role by doing just this. Throughout, developing and fostering a relationship between societies built upon respect and mutual benefit is essential to ensuring productive and sustainable crosscollaborations.

\section{The Role of Global Governance}

The WFNS is a professional, nongovernmental organization representing 118 individual societies of neurosurgery worldwide. With the overarching purpose to promote global improvement in neurosurgical care, capacity building through education and surgeon training is among the defining principles of the Federation. For example, as the glaring shortage of neurosurgeons in Africa has grown, the WFNS has taken specific actions such as facilitation of the "Africa 100" initiative championed by Professor Majid Samii and designed to increase the number of permanent practicing surgeons on the continent. Via such initiatives, the Federation can work to establish educational paradigms that incorporate open-access, web-based educational platforms accessible to trainees and consultants from all corners of the globe. Indeed, the WFNS already has a track record as a central, nonpartisan organization for open collaboration between member partners and the transfer of ideas and technologies across the many national societies. Moving forward, the WFNS is optimally suited for advocacy toward and coordination of the neurosurgery capacity building effort. In so doing, the Federation is well positioned to: 1) raise awareness of the unmet neurosurgical care as a global public health issue; 2 ) develop educational and practice guidelines based upon member input; 3) collect and monitor global neurosurgical workforce and capacity data; and 4) facilitate coordination and collaboration between the academic community and state-sponsored agencies toward efficiently addressing the worldwide deficit in neurosurgical care.
Integrating Neurosurgery Within the Overall Surgical Community

Neurosurgical efforts aimed at LMICs would benefit greatly from integration with ongoing global surgical programs through partnerships with professional societies, academia, and nongovernmental organizations, to avoid duplication and to complement the surgical system-strengthening efforts of each stakeholder. In many regions, there already exist efforts designed to develop or improve surgical and anesthesia service delivery, enhancing the overall surgical ecosystem. Consider, for example, the essential nature of a blood bank for safe surgical care: it matters little if packed red blood cells are used for a postpartum hemorrhage, a malaria crisis, or a severe TBI. Surgeons of all types and anesthetists can work together synergistically to develop and maintain components of the entire surgical delivery system. Partnership with United Nations bodies, such as the WHO, provide collaborative arrangements with local governments that offer the advantage of enhancing service delivery mechanisms incountry. The WHO, through the Emergency and Essential Surgical Care Program, is poised to facilitate global coordination of neurosurgical efforts along with other surgical stakeholders as defined by the mandates of member states.

Currently, combined efforts targeting advocacy, service delivery, data collection, essential medicines, and health workforce are critical, thus fulfilling the mandates of World Health Assembly Resolution 68.15 (2015) on strengthening emergency and essential surgical care and anesthesia as a component of universal health coverage? all with direct links to the sustainable development goals. ${ }^{18}$

\section{Strengths and Limitations of the Study}

The estimates and conclusions outlined in this summary must be considered in the context of several limitations. First, most disease volume figures derive from large systematic reviews and meta-analyses, as reliable, population-based estimates for each country or region are nonexistent. Similarly, the surgical workforce figures relied upon accurate neurosurgical association reporting, and at times questionnaire responses and statistical modeling, all of which carry bias and intrinsic inconsistencies. Throughout the tables and text, we assume that neurosurgeons are optimally distributed geographically in relation to the at-risk population. We also assume that each trained neurosurgeon is capable of safely treating each condition encompassed within the designation of essential neurosurgical care. While these assumptions are generally safe for epidemiological modeling at the region- or country-level, they would not permit accurate allocation of resources at the city or hospital level. In relation to surgical capacity, we have focused on the surgeon only-not the wealth of infrastructure, equipment, and personnel required alongside the surgeon to facilitate safe and timely provision of neurosurgical care. We acknowledge the tremendous importance of these resources, and we are actively conducting a worldwide survey to better understand their accessibility to current and future surgeons. ${ }^{14}$

In an effort to prioritize the most life-threatening neurosurgical conditions worldwide, we have deliberately excluded many important conditions commonly addressed by neurosurgeons, most notably degenerative spine dis- 
ease. This relative limitation warrants specific elaboration. In the US, with more than 5000 neurosurgeons, we estimate the case capacity is nearly 1.2 million cases/year. Meanwhile, the volume of essential neurosurgical cases is estimated at only 576,000, suggesting a surplus of neurosurgeons exists in the US. We would caution the reader against this conclusion. The majority of neurosurgical cases performed in the US are for conditions falling outside the designation used in this report of essential neurosurgical care. Indeed, entities like degenerative spine disease, chronic pain, movement disorders, and many other conditions are well known to cause disability and suffering if left untreated. Thus, a shortage of neurosurgeons may exist even in the most developed of countries, depending upon the level and type of neurosurgical care demanded by a given population. The scope and purpose of this paper is to offer a description of the workforce capacity and deficit that exists for essential neurosurgical care, a designation that itself aims to prioritize those patients who are most likely to benefit from lifesaving and disabilityaverting neurosurgical care.

Finally, this report ignores the concept of task sharing and the number of procedures potentially performed by nonneurosurgeon providers. Training general surgeons in common neurosurgical procedures such as ventriculoperitoneal shunt insertion and burr hole craniotomy is a practical strategy that might more quickly help curb the overwhelming neurosurgical burden. Reasonable neurosurgical experts will disagree on the ethics and qualityrelated implications of this frequently used strategy in the realm of global health. The potential capacity-related consequences of this strategy, however, are absent from the figures provided above.

While inherent limitations exist for an epidemiological survey and capacity estimation of this scope, careful and deliberate measures were taken to ensure data validity and provide methodological transparency throughout. Rather than relying on surgical log data from hospitals and health systems where the surgical care is known to be inadequate, we approached surgical deficit figures first from the disease component of the equation. Though data modeling was necessary in some cases, the disease-specific estimates represent the best available evidence to date, derived from among the most comprehensive systematic reviews available in the neurosurgical literature. Additionally, surgical provider data arose from the most far-reaching effort yet to quantify the worldwide population of neurosurgeons, and involved a partnering effort with many active international neurosurgery initiatives such as the WFNS.

\section{Conclusions}

Each year, 13.8 million new essential neurosurgical operative cases and 22.6 million new consultative cases present worldwide. TBI, tumor, hydrocephalus, and strokerelated conditions constitute the majority of essential neurosurgical volume globally. Regional variation in disease incidence is likely influenced by a host of factors including baseline population demographics, risk factor exposure, and genetic susceptibility. Wide workforce gaps exist, and the uneven distribution of neurosurgeons to disease volume creates large geographic pockets of surgical access shortage. Nearly 23,000 additional neurosurgeons are needed in low- and middle-income countries to adequately address the unmet deficit of more than 5 million neurosurgical operations worldwide.

\section{Acknowledgments}

We would like to thank Ron Baticulon, MD, for his graphical expertise. The work in this report was enhanced by the logistical, financial, or in-kind support that we received from the following organizations: Boston Children's Hospital Global Hydrocephalus and Spina Bifida Fund, Harvard Medical School Department of Global Health and Social Medicine, John D. and Catherine T. MacArthur Foundation, Kellogg Institute for International Studies at the University of Notre Dame, Vanderbilt Medical Scholars Program, Vanderbilt University Medical Center, and the WFNS.

\section{Appendix \\ Collaborators}

We would like to thank the following individuals for their dedication and contribution to identifying the global neurosurgical deficit. Collaborators are listed in alphabetical order:

Amos O. Adeleye, MBBS, Amit Agrawal, MCh, Blake C. Alkire, MD, MPH, Julia R. Amundson, BS, Hildo Azevedo-Filho, $\mathrm{MD}, \mathrm{PhD}$, Ronnie E. Baticulon, MD, Joseph S. Bell, PhD, Erica Bisson, MD, MPH, Kamila M. Bond, BA, Vivek P. Buch, MD, Matthew C. Davis, MD, Robert Dempsey, MD, Serena Faruque, MS, $\mathrm{PhD}$, Laurence Glancz, MBBS, BSc, William B. Gormley, MD, MPH, MBA, Saksham Gupta, BA, Michael M. Haglund, MD, PhD, MACM, Roger Härtl, MD, Joshua D. Hughes, MD, Ya Ching Hung, MD, Yoko Kato, MD, PhD, Robert M. Koffie, MD, PhD, Ramesh Kumar, MD, Jacob R. Lepard, MD, Jaims Lim, BS, Muhammad Raji Mahmud, MD, John G. Meara, MD, DMD, MBA, Rania A. Mekary, MSc, PhD, Basant K Misra, MD, Jacques J. Morcos, MD, Swagoto Mukhopadhyay, MD, Brian V. Nahed, MD, MSc, Enrique Osorio-Fonseca, MD, Sophie Peeters, BS, Maria Punchak, MSc, Mahmood M. Qureshi, MD, Christian Lopez Ramos, BS, Vijay Ravindra, MD, MSPH, Faith C. Robertson, BS, Jeffrey V. Rosenfeld, MD, Gail Rosseau, MD, Andrés M. Rubiano, MD, Sonal Sachdev, MD, Steven S. Senglaub, MS, Salman Y. Sharif, MD, Mark G. Shrime, MD, MPH, PhD, Kerry A. Vaughan, MD, Eka J. Wahjoepramono, MD, PhD, John C. Wellons III, MD, MSPH, and Ismaeel Yunusa, PharmD.

\section{References}

1. Albright AL: Reflections on developing pediatric neurosurgery in Sub-Saharan Africa. J Neurosurg Pediatr 18:127138,2016

2. Andrews RJ, Quintana LM: Neurosurgical care for oneneurosurgical care for all: global neurosurgical care has global benefits! World Neurosurg 85:22-24, 2016

3. Bell JS, Koffie R, Rattani A, Dewan MC, Baticulon R, Qureshi M, et al: Global incidence of brain and spinal tumors by geographic region and income level, presented at the Congress of Neurological Surgeons Annual Meeting, Boston, 2017 (Poster) (http://2017.cns.org/posterbrowser.aspx) [Accessed April 16, 2018]

4. Debas HT, Donkor P, Gawande AA, Kruk M, Jamison DT, Mock C, et al: Disease Control Priorities: Essential Surgery, ed 3. Washington, DC: World Bank Publications, 2015

5. Dewan MC, Rattani A, Baticulon RE, Faruque S, Johnson WD, Dempsey RJ, et al: The operative proportion of neurosurgical disease worldwide: an estimation from the surgeon perspective. J Neurosurg [in press], 2018

6. Dewan MC, Rattani A, Gupta S, Baticulon RE, Hung YC, 
Punchak M, et al: Estimating the global incidence of traumatic brain injury. J Neurosurg [in press], 2018

7. Dewan MC, Rattani A, Mekary RA, Glancz LJ, Yunusa I, Baticulon RE, et al: Global hydrocephalus epidemiology and incidence: systematic review and meta-analysis. J Neurosurg [in press], 2018

8. Hughes JD, Bond KM, Mekary RA, Dewan MC, Rattani A, Kato Y, et al: A systematic literature review for the global incidence of central nervous system vascular lesions and meta-analysis of hemorrhagic aneurysms, arteriovenous malformations, and dural arteriovenous fistulas, presented at the Congress of Neurological Surgeons Annual Meeting, Boston, 2017 (Poster) (http://2017.cns.org/posterbrowser.aspx) [Accessed January 12, 2018]

9. Hughes JD, Bond K, Mekary R, Dewan MC, Rattani A, Kato $\mathrm{Y}$, et al: Estimating the global incidence of central nervous system vascular lesions: a systematic review and meta-analysis of aneurysmal subarachnoid hemorrhage. World Neurosurg [in press], 2018

10. Kancherla V, Walani SR, Weakland AP, Bauwens L, Oakley GP Jr, Warf BC: Scorecard for spina bifida research, prevention, and policy - a development process. Prev Med 99:1320, 2017

11. Kumar R, Lim J, Mekary RA, Rattani A, Dewan MC, Sharif SY, et al: Traumatic spinal injury: global epidemiology and worldwide volume. World Neurosurg [epub ahead of print], 2018

12. Meara JG, Leather AJM, Hagander L, Alkire BC, Alonso N, Ameh EA, et al: Global Surgery 2030: evidence and solutions for achieving health, welfare, and economic development. Lancet 386:569-624, 2015

13. Park KB, Johnson WD, Dempsey RJ: Global neurosurgery: the unmet need. World Neurosurg 88:32-35, 2016

14. Punchak M, Mukhopadhyay S, Sachdev S, Hung YC, Peeters S, Rattani A, et al: Neurosurgical care: availability and access in low-income and middle-income countries. World Neurosurg 112:e240-e254, 2018

15. Ravindra V, Senglaub S, Rattani A, Park KB, Dewan MC, Shrime MG: Degenerative lumbar spine disease: estimating global incidence and worldwide volume. Global Spine J [in press], 2018

16. Robertson FC, Lepard JR, Davis MC, Mekary R, Yunusa I, Dewan MC, et al: Epidemiology of CNS infectious disease: a meta-analysis and implications for the global neurosurgeon. $\mathbf{J}$ Neurosurg [in press], 2018

17. Shrime MG, Bickler SW, Alkire BC, Mock C: Global burden of surgical disease: an estimation from the provider perspective. Lancet Glob Health 3 (Suppl 2):S8-S9, 2015
18. United Nations: Transforming Our World: the 2030 Agenda for Sustainable Development. (https://sustainabledevelopment.un.org/post2015/ transformingourworld) [Accessed January 12, 2018].

19. Vaughan KA, Ramos CL, Buch VP, Mekary R, Amundson JR, Shah M, et al: An estimation of global volume of surgically treatable epilepsy based on a systematic review and meta-analysis of epilepsy. J Neurosurg [in press], 2018

20. Warf BC: "Who is my neighbor?" Global neurosurgery in a non-zero-sum world. World Neurosurg 84:1547-1549, 2015

21. World Health Organization: World Health Assembly resolution 68.15: Strengthening emergency and essential surgical care and anaesthesia as a component of universal health coverage. (http://apps.who.int/gb/ebwha/pdf_files/WHA68/ A68_R15-en.pdf) [Accessed January 12, 2018]

\section{Disclosures}

The authors report no conflict of interest concerning the materials or methods used in this study or the findings specified in this paper.

\section{Author Contributions}

Conception and design: Dewan. Acquisition of data: Dewan, Rattani. Analysis and interpretation of data: Dewan, Rattani, Fieggen, Arraez, Servadei, Johnson, Warf. Drafting the article: Dewan, Fieggen, Arraez, Servadei, Boop, Johnson, Warf. Critically revising the article: all authors. Reviewed submitted version of manuscript: all authors. Approved the final version of the manuscript on behalf of all authors: Dewan. Statistical analysis: Rattani. Administrative/technical/material support: Rattani.

\section{Supplemental Information Online-Only Content}

Supplemental material is available with the online version of the article.

Appendix. https://thejns.org/doi/suppl/10.3171/2017.11. JNS171500.

\section{Correspondence}

Michael C. Dewan: Vanderbilt University Medical Center, Nashville, TN.dewan.michael@gmail.com. 